\title{
Parallel Treatment of Estimation of SU(2) and Phase Estimation ${ }^{1}$
}

\author{
Masahito Hayashi \\ Quantum Computation and Information Project, ERATO, JST \\ 5-28-3, Hongo, Bunkyo-ku, Tokyo, 113-0033, Japan
}

\begin{abstract}
We discuss the accuracy of the estimation of the $n$ identical unknown actions of $\mathrm{SU}(2)$ by using entanglement. This problem has a similar structure with the phase estimation problem, which was discussed by Bužek, Derka, and Massar[1]. The estimation error asymptotically decreases to zero with an order of $\frac{1}{n^{2}}$ at least.
\end{abstract}

Key words: SU(2), Estimation, Entanglement, Phase estimation PACS: 03.65.Wj, 03.65.Ud, 02.20.-a

\section{Introduction}

\subsection{Estimation of unitary action}

Quantum information processing architecture is constructed with a combination of various quantum devices. In other words, each quantum device contributes towards obtaining the output quantum state from the input quantum state. Hence, it is necessary to identify the quantum operation acting on each device. This problem is referred to as the identification of the quantum operation; this problem usually entails the estimation of the operation based on the pair of the input (initial) state and the data of the measurement for the output

Email address: masahito@qci.jst.go.jp (Masahito Hayashi).

1 The material in this paper was presented in part at The Ninth Quantum Information Technology Symposium (QIT9), Atsugi, Kanagawa, Japan, December 11-12, 2003. It was also presented in part at The Seventh International Conference on Quantum Communication, Measurement and Computing, Glasgow, UK, July 2529, 2004. The final section (Concluding remark) was added after these presentations. 
state. Generally, such a quantum operation is described by a trace-preserving completely positive (TP-CP) map. In this paper, we consider a noiseless case in which no noise appears in the quantum operation. In this case, the quantum operation is described by a unitary matrix. The adiabatic case is also treated in a similar manner.

When the reference system is unitarily equivalent to the input system and the initial state is a maximally entangled state in a joint system between the original input system and the reference system, the final state in the joint system is the maximally entangled state described by the unitary matrix acting on the input system. Hence, by repeating this operation $n$ times, we can estimate this unitary matrix by performing appropriate measurement for the total joint system between output system and the reference system. In the two-dimensional case, Fujiwara [2] showed that a maximally entangled state is the optimal initial state in the composite system between the single input system and its reference system. Ballester [3] discussed this problem in the $d$ dimensional case. When their method is applied to the case of $n$ applications of this unknown operation, the estimation error decreases to zero only with order $\frac{1}{n}$; this is the case even if the output measurement is optimized. This order is obtained from the accuracy of state estimation by using an identical state preparation. Therefore, it is worthwhile to consider the possibility of further improvement.

\subsection{One-parameter case: Phase estimation}

The phase estimation problem is closely related to the problem discussed in this paper. In the phase estimation problem, we estimate the eigenvalue $e^{i \theta}$ of the unknown unitary matrix acting on a two-level system when we know its eigenvectors. The phase estimation with the fixed input state was discussed by Helstrom[4] first. Holevo [5] extended Helstrom's result to a more general framework of group covariance. The optimization problem of the input state for phase estimation was discussed by Bužek et al. [1] in an asymptotic setting. They proved that the error decreases to zero with speed $\frac{\pi^{2}}{4 n^{2}}$ when we choose the optimal input state and optimal measurement. However, the error goes to

zero with the order $\frac{1}{n}$ in the usual statistical parameter estimation. Therefore, this unexpected result indicates the importance of the entangled input state.

\subsection{Three-parameter case: Our result}

In this study, we discuss whether such a phenomenon occurs in the estimation of $\mathrm{SU}(2)$ unitary action. In this paper, we adopt the error function $d(U, \hat{U}) \stackrel{\text { def }}{=}$ 
$1-\left|\operatorname{Tr} \frac{U^{-1} \hat{U}}{2}\right|^{2}$ between the true SU(2) matrix $U$ and the estimated matrix $\hat{U}$. Then, we obtain an unexpected relation between our problem and that discussed by Bužek et al.[1]. Thanks to this relation, we can show that the error decreases to zero in proportion to $\frac{1}{n^{2}}$ at least. Further, the coefficient is equal to $\pi^{2}$ if the estimator is constructed based on this relation. It is shown that this bound can be asymptotically attained with no use of the reference system. Instead of use of the reference system, we regard a part of the composite system of $n$ input systems as the tensor product of the system of interest and the reference system. In other words, an effect of "self-entanglement" is used in this method.

\section{Phase estimation: Estimation of eigenvalues with the knowledge of eigenvectors}

In order to demonstrate the interesting relation between phase estimation and estimation of $\mathrm{SU}(2)$ action, we briefly summarize the fact known with regard to phase estimation. When the eigenvectors of the unknown $\mathrm{SU}(2)$ matrix $U$ are known, the estimation problem can be reduced to the estimation of the unknown parameter $\theta \in[0,2 \pi)$ of the family $\left\{U_{\theta} \stackrel{\text { def }}{=}\left(\begin{array}{cc}e^{i \theta} & 0 \\ 0 & 1\end{array}\right) \mid \theta \in[0,2 \pi)\right\}$ because the action of $U$ is equivalent to that of $c U$.

In our framework, we assume that the tensor product matrix $U_{\theta}^{\otimes n}$ acts in the tensor product space $\mathcal{H}^{\otimes n}$ and that we can select an arbitrary initial state in the tensor product space $\mathcal{H}^{\otimes n}$. That is, we can select the input state $x \in \mathcal{H}^{\otimes n}$ and the estimating POVM $M^{n}(d \hat{\theta})$ on $\mathcal{H}^{\otimes n}$ with outcomes in $[0,2 \pi)$. When we evaluate the error between the true parameter $\theta$ and the estimated parameter $\hat{\theta}$ by the function $\sin ^{2} \frac{\theta-\hat{\theta}}{2}$, the mean error is given by

$$
\mathcal{D}_{\theta}^{n}\left(M^{n}, x\right) \stackrel{\text { def }}{=} \int_{0}^{2 \pi} \sin ^{2} \frac{\theta-\hat{\theta}}{2}\left\langle x\left|\left(U_{\theta}^{\otimes n}\right)^{\dagger} M^{n}(d \hat{\theta}) U_{\theta}^{\otimes n}\right| x\right\rangle .
$$

Thus, when we fix the input state $x \in \mathcal{H}^{\otimes n}$, our estimation problem can be reduced to the estimation with the state family $\left\{U_{\theta}^{\otimes n}|x\rangle\left\langle x\left|\left(U_{\theta}^{\otimes n}\right)^{\dagger}\right| \theta \in[0,2 \pi)\right\}\right.$.

In order to treat this problem in a simple manner, we focus on the phase estimation problem in the $d$-dimensional space $\mathcal{H}^{\prime}$ spanned by the orthonormal basis $u_{1}, \ldots, u_{d}$ as follows. We select a vector $x=\left(x_{k}\right)_{k=1}^{d}=\sum_{k=1}^{d} x^{k} u_{k}$ satisfying 


$$
\sum_{k=1}^{d}\left|x_{k}\right|^{2}=1
$$

Suppose that the state to be estimated has the form $\rho_{\theta, x} \stackrel{\text { def }}{=} U_{\theta}|x\rangle\langle x| U_{\theta}^{*}$ with the unknown parameter $\theta \in[0,2 \pi)$ and the unitary matrix $U_{\theta} \stackrel{\text { def }}{=} \sum_{k=1}^{d} e^{i k \theta}\left|u_{k}\right\rangle\left\langle u_{k}\right|$. In this case, the error of the estimator $M(d \hat{\theta})$ can be be expressed as

$$
\mathcal{D}_{\theta}(M, x) \stackrel{\text { def }}{=} \int_{0}^{2 \pi} \sin ^{2} \frac{\theta-\hat{\theta}}{2} \operatorname{Tr} M(d \hat{\theta}) \rho_{\theta, x} .
$$

In order to ensure accuracy, it is appropriate to focus on the worst case. In other words, we minimize the worst error $\max _{\theta} \mathcal{D}_{\theta}(M, x)$. This problem is refereed to as minimax.

On the other hand, since the state $\rho_{\theta, x}$ has symmetry

$$
\rho_{\theta+\theta^{\prime}, x}=U_{\theta^{\prime}} \rho_{\theta, x} U_{\theta^{\prime}}^{\dagger}
$$

it is natural to treat the measurement $M(\hat{\theta}) d \hat{\theta}$ with the same symmetry:

$$
M\left(\hat{\theta}+\hat{\theta}^{\prime}\right)=U_{\hat{\theta}^{\prime}} M(\hat{\theta}) U_{\hat{\theta}^{\prime}}^{\dagger}
$$

A measurement $M$ satisfying (4) is referred to as the covariant measurement and it has the form

$$
M^{T}(d \hat{\theta}) \stackrel{\text { def }}{=} U_{\hat{\theta}} T U_{\hat{\theta}}^{\dagger} \frac{d \hat{\theta}}{2 \pi}
$$

where the Hermitian matrix $T=\sum_{k, l} t_{k, l}\left|u_{k}\right\rangle\left\langle u_{l}\right|$ satisfies

$$
T \geq 0 \text { and } t_{k, k}=1 \text {. }
$$

Holevo proved that the minimum error in the minimax criteria is equal to the minimum error among covariant measurements, i.e.,

$$
\min _{M} \max _{\theta} \mathcal{D}_{\theta}(M, x)=\min _{M: \text { covariant }} \mathcal{D}_{\theta}(M, x) .
$$

This relation is referred as the quantum Hunt-Stein lemma, and was proved in a more general covariant setting[5].

By using elementary formulas of trigonometric functions, the equation 


$$
=\begin{gathered}
\int_{0}^{2 \pi} \sin ^{2} \frac{\theta-\hat{\theta}}{2} \operatorname{Tr} U_{\theta}\left|u_{k^{\prime}}\right\rangle\left\langle u_{l^{\prime}}\left|U_{\theta}^{\dagger} U_{\hat{\theta}}\right| u_{l}\right\rangle\left\langle u_{k}\right| U_{\hat{\theta}}^{\dagger} \frac{d \hat{\theta}}{2 \pi} \\
\delta_{k, k^{\prime}} \delta_{l, l^{\prime}}\left(\frac{1}{2} \delta_{k, l}-\frac{1}{4} \delta_{k, l-1}-\frac{1}{4} \delta_{k-1, l}\right)
\end{gathered}
$$

can be verified. By using this relation, we have

$$
\begin{gathered}
\mathcal{D}_{\theta}\left(M_{T}, x\right)=\frac{1}{2} \sum_{k=1}^{d}\left|x_{k}\right|^{2} t_{k, k}-\frac{1}{4} \sum_{k=1}^{d-1}\left(\overline{x_{k}} x_{k+1} t_{k+1, k}+\overline{x_{k+1}} x_{k} t_{k, k+1}\right) \\
\geq \frac{1}{2} \sum_{k=1}^{d}\left|x_{k}\right|^{2}-\frac{1}{4} \sum_{k=1}^{d-1}\left(\left|\overline{x_{k}} x_{k+1}\right|+\left|\overline{x_{k+1}} x_{k}\right|\right)=\frac{1}{2}\left(1-\sum_{k=1}^{d-1}\left|x_{k}\right|\left|x_{k+1}\right|\right)
\end{gathered}
$$

where the inequality follows from $\left|\left\langle u_{k}|T| u_{k+1}\right\rangle\right| \leq \sqrt{\left\langle u_{k}|T| u_{k}\right\rangle\left\langle u_{k+1}|T| u_{k+1}\right\rangle}=$ 1. Hence, the equality holds iff

$$
t_{k, k+1}=\frac{\overline{x_{k}} x_{k+1}}{\left|x_{k}\right|\left|x_{k+1}\right|}
$$

Since the matrix $T_{x} \stackrel{\text { def }}{=} \sum_{k, l} \frac{\overline{x_{k}} x_{l}}{\left|x_{k}\right|\left|x_{l}\right|}\left|u_{k}\right\rangle\left\langle u_{l}\right|$ satisfies the conditions (6) and (9), we obtain

$$
\min _{M: \text { covariant }} \mathcal{D}_{\theta}(M, x)=\frac{1}{2}\left(1-\sum_{k=1}^{d-1}\left|x_{k}\right|\left|x_{k+1}\right|\right) \text {. }
$$

Now, we consider the estimation of the unknown unitary $U_{\theta}$ with its multiple actions. Since the unitary matrix $U_{\theta}^{\otimes n}$ has eigenvalues $1, e^{i \theta}, \ldots, e^{n i \theta}$, the application of the relation (10) to the $d=n+1$ case yields

$$
\begin{aligned}
\mathcal{D}_{o p t}^{n} & \stackrel{\text { def }}{=} \min _{x \in \mathcal{H}^{\otimes n}} \min _{M^{n}} \max _{\theta} \mathcal{D}_{\theta}^{n}\left(M^{n}, x\right) \\
& =\min _{\left(a_{k}\right)_{k=0}^{n}}\left\{\frac{1}{2}\left(1-\sum_{k=1}^{n} a_{k} a_{k-1}\right) \mid \sum_{k=0}^{n} a_{k}^{2}=1, a_{k} \geq 0\right\} .
\end{aligned}
$$

In this problem, the eigenspace $\mathcal{H}_{k}$ is not one-dimensional for the eigenvalue $e^{i k \theta}$. Hence, any initial state can be written as $\sum_{k} x_{k} e^{k}$, where $e^{k}$ is a normalized vector in $\mathcal{H}_{k}$. Therefore, the minimum error can be expressed in the above form. Bužek, et al.[1] proved that the minimum error is attained by the coefficient $a_{k}=\frac{\sqrt{2}}{\sqrt{n+1}} \sin \frac{\pi(k+1 / 2)}{n+1}$ and is almost equal to $\frac{\pi^{2}}{4 n^{2}}$, i.e.,

$$
\mathcal{D}_{o p t}^{n} \cong \frac{\pi^{2}}{4 n^{2}}
$$


In this setting, the irreducible representation space of the action of the onedimensional circle $S^{1}=[0,2 \pi)$ is the one-dimensional space spanned by $u_{k}$. In the above discussion, we use $n+1$ different irreducible representation spaces.

\section{Estimation of the unknown $\mathrm{SU}(2)$ action}

Next, we proceed to the estimation of the unknown $\mathrm{SU}(2)$ action $g \in \mathrm{SU}(2)$ in the two-dimensional space $\mathcal{H}$. In this problem, the unitary matrix $g^{\otimes n}$ acts in the tensor product space $\mathcal{H}^{\otimes n}$, and a suitable initial state in $\mathcal{H}^{\otimes n}$ can be selected for this estimation. On the other hand, Fujiwara[2] focused on the estimation problem where the initial state is entangled with the reference system $\mathcal{H}_{R}$, in which the unknown $\mathrm{SU}(2)$ action $g$ does not act. He proved that this method reduces the estimation error. However, he did not treat the entanglement between the $n$-tensor product space $\mathcal{H}^{\otimes n}$ and its reference system.

Now, we consider the amount of the estimation error can be reduced by use of entanglement among tensor product space. In other words, our framework has a wider choice. In our problem, we can select an initial state entangled between $\mathcal{H}^{\otimes n}$ and the reference space $\mathcal{H}_{R}^{\otimes n}$ that is unitarily equivalent to the original input system $\mathcal{H}^{\otimes n}$. Hence, when we select the initial state $x$ on the tensor product space $\mathcal{H}^{\otimes n} \otimes \mathcal{H}_{R}^{\otimes n}$ and the measurement (POVM) $M^{n}(d \hat{g})$ in $\mathcal{H}^{\otimes n} \otimes \mathcal{H}_{R}^{\otimes n}$ with the outcome in $\mathrm{SU}(2)$, the error is evaluated as

$$
\mathcal{D}_{g}(M, x) \stackrel{\text { def }}{=} \int_{\mathrm{SU}(2)} d(g, \hat{g})\left\langle x\left|\left(g^{\otimes n} \otimes I\right)^{*} M(d \hat{g})\left(g^{\otimes n} \otimes I\right)\right| x\right\rangle .
$$

Here, we focus on the $\mathrm{SU}(2)$ action on the tensor product space $\mathcal{H}^{\otimes n}$. Its irreducible decomposition is given as follows:

$$
\begin{aligned}
\mathcal{H}^{\otimes 2 d} & =\bigoplus_{k=0}^{d} \mathcal{H}_{2 k+1} \otimes \mathcal{H}_{2 d, 2 k+1} \\
\mathcal{H}^{\otimes 2 d-1} & =\bigoplus_{k=1}^{d} \mathcal{H}_{2 k} \otimes \mathcal{H}_{2 d-1,2 k},
\end{aligned}
$$

where $\mathcal{H}_{k}$ is the $k$-dimensional irreducible space of the $\mathrm{SU}(2)$ action, and $\mathcal{H}_{n, k}$ is the corresponding irreducible space of the action of the permutation group, where $\mathrm{SU}(2)$ does not act. Note that the dimension of $\mathcal{H}_{n, k}$ is equal to the number of representation spaces equivalent to $\mathcal{H}_{k}$ in the tensor product space $\mathcal{H}^{\otimes n}$. Hereafter, we denote the $\mathrm{SU}(2)$ action on $\mathcal{H}_{k}$ by $V_{g}^{k}$. 
For simplicity, first we focus on the estimation of the $\mathrm{SU}(2)$ action in a single irreducible space $\mathcal{H}_{j}$. Now, we select the initial state as the maximally entangled state $x_{E}^{j}$ between $\mathcal{H}_{j}$ and the reference space $\mathcal{H}_{j, R}$ that is unitarily equivalent with the space $\mathcal{H}_{j}$. The measurement is selected as the POVM $M_{j, E}(d \hat{g}) \stackrel{\text { def }}{=} j^{2} V_{\hat{g}}^{j} \otimes I\left|x_{E}^{j}\right\rangle\left\langle x_{E}^{j}\right|\left(V_{\hat{g}}^{j} \otimes I\right)^{\dagger} \mu(d \hat{g})$, where $\mu$ is the invariant probability distribution on $\mathrm{SU}(2)$ and the integer $j^{2}$ is the normalizing factor. By using Schur's lemma, we can easily verify that the total integral is constant times the identity matrix because the state $x_{E}^{j}$ is maximally entangled. Since $\left(\operatorname{Tr} V_{g}^{j}\right)^{*}=\operatorname{Tr}\left(V_{g}^{j}\right)^{\dagger}$, the average error is calculated as

$$
\begin{aligned}
& \int_{\mathrm{SU}(2)} d(g, \hat{g}) j^{2}\left\langle x_{E}^{j}\left|\left(V_{g}^{j}\right)^{\dagger} V_{\hat{g}}^{j}\right| x_{E}^{j}\right\rangle\left\langle x_{E}^{j}\left|\left(V_{\hat{g}}^{j}\right)^{\dagger} V_{g}^{j}\right| x_{E}^{j}\right\rangle \mu(d \hat{g}) \\
= & \int_{\mathrm{SU}(2)} d(g, \hat{g})\left|\operatorname{Tr} V_{\hat{g}^{-1} g}^{j}\right|^{2} \mu(d \hat{g})=\int_{\mathrm{SU}(2)} d(I, \hat{g})\left|\operatorname{Tr} V_{\hat{g}^{-1}}^{j}\right|^{2} \mu(d \hat{g}) \\
= & \int_{\mathrm{SU}(2)} d(I, \hat{g})\left|\operatorname{Tr} V_{\hat{g}}^{j}\right|^{2} \mu(d \hat{g})=\left\{\begin{array}{l}
\frac{3}{4} j=1 \\
\frac{1}{2} j \geq 2 .
\end{array}\right.
\end{aligned}
$$

The final equation follows from the elementary calculus of trigonometric functions. This calculation seems to indicate that if we use only one irreducible space $\mathcal{H}_{j}$ and even if its dimension is large, the estimation error cannot be reduced $^{2}$. Hence, in order to reduce the estimation error, it may be needed to use several irreducible spaces.

Hereafter, we consider the case of $n=2 d-1$; however, the following discussion can be applied to the even case. In the odd case, $d$ distinct irreducible spaces exist. Hence, it is essential to use the correlation between them. We investigate the following subspace of $\mathcal{H}^{\otimes(2 d-1)} \otimes \mathcal{H}_{R}^{\otimes(2 d-1)}$ :

$$
\bigoplus_{k=1}^{m} \mathcal{H}_{2 k} \otimes \mathcal{H}_{2 k, R}
$$

Further, we denote the $\mathrm{SU}(2)$ representation on this space by $U_{g}^{2 d-1}$.

As demonstrated later, this problem can be treated parallel to the phase estimation in which the base $u_{j}$ corresponds to the maximally entangled state $x_{E}^{2 k}$ or the vector $(2 k) x_{E}^{2 k}$. We select a vector $\vec{x}_{d}=\left(x_{k}\right)_{k=1}^{d}$ that satisfies the condition (1), and let the initial state be $x_{\vec{x}_{d}}^{2 d-1} \stackrel{\text { def }}{=} \bigoplus_{k=1}^{d} x_{k} x_{E}^{2 k}$. In a manner similar to (5), the measurement is selected as

$\overline{2}$ This fact has been proved by Chiribella et al.[16] after the submission of the preliminary version of this paper. That is, they proved the optimality of this POVM in a more general framework. 


$$
M_{2 d-1}^{T}(d \hat{g}) \stackrel{\text { def }}{=} U_{\hat{g}}^{2 d-1} \sum_{k, l} t_{k, l}\left|(2 k) x_{E}^{2 k}\right\rangle\left\langle(2 l) x_{E}^{2 l}\right|\left(U_{\hat{g}}^{2 d-1}\right)^{\dagger} \mu(d \hat{g})
$$

based on a Hermitian matrix $T=\left(t_{k, j}\right)$ satisfying condition (6). Using Schur's lemma and condition (6), we can verify that its total integral is an identity matrix. In a manner similar to $(7)$, the equations

$$
\begin{aligned}
& \int_{\mathrm{SU}(2)} d(g, \hat{g}) \operatorname{Tr} U_{g}^{2 d-1}\left|x_{E}^{2 k^{\prime}}\right\rangle\left\langle x_{E}^{2 l^{\prime}}\left|\left(U_{g}^{2 d-1}\right)^{\dagger} U_{\hat{g}}^{2 d-1}\right|(2 k) x_{E}^{2 k}\right\rangle\left\langle(2 l) x_{E}^{2 l}\right|\left(U_{\hat{g}}^{2 m-1}\right)^{\dagger} \mu(d \hat{g}) \\
= & \delta_{k, k^{\prime}} \delta_{l, l^{\prime}} \int_{\mathrm{SU}(2)} d(g, \hat{g}) \operatorname{Tr} V_{\hat{g}}^{2 k}\left(\operatorname{Tr} V_{\hat{g}}^{2 l}\right)^{\dagger} \mu(d \hat{g})=\delta_{k, k^{\prime}} \delta_{l, l^{\prime}}\left(\frac{1}{2} \delta_{k, l}-\frac{1}{4} \delta_{k, l-1}-\frac{1}{4} \delta_{k-1, l}\right)
\end{aligned}
$$

holds, where the final equation is derived in section 4 based on elementary formulas of trigonometric functions. By using this relation in a manner similar to (8), we have

$$
\begin{aligned}
& \int_{\mathrm{SU}(2)} d(g, \hat{g}) \operatorname{Tr} U_{g}^{2 d-1}\left|x_{\vec{x}_{d}}^{2 d-1}\right\rangle\left\langle x_{\vec{x}_{d}}^{2 d-1}\right|\left(U_{g}^{2 d-1}\right)^{\dagger} M_{2 d-1}^{T}(d \hat{g}) \\
= & \frac{1}{2} \sum_{k=1}^{d}\left|x_{k}\right|^{2} t_{k, k}-\frac{1}{4} \sum_{k=1}^{d}\left(\overline{x_{k-1}} x_{k} t_{k, k-1}+\overline{x_{k}} x_{k-1} t_{k-1, k}\right) \\
\geq & \frac{1}{2} \sum_{k=1}^{d}\left|x_{k}\right|^{2}-\frac{1}{4} \sum_{k=1}^{d-1}\left(\left|\overline{x_{k}} x_{k+1}\right|+\left|\overline{x_{k+1}} x_{k}\right|\right)=\frac{1}{2}\left(1-\sum_{k=1}^{d-1}\left|x_{k}\right|\left|x_{k+1}\right|\right) .
\end{aligned}
$$

The equality holds when matrix $T=\left(t_{k, l}\right)$ satisfies $(9)$. Thus, the optimal error of this estimation method coincides with $\mathcal{D}_{\text {opt }}^{m-1}$. That is, our problem is reduced to phase estimation problem by Bužek et al. When we select a suitable initial state and measurement in the case of large $n$, the estimation error is equal to $\frac{\pi^{2}}{n^{2}}$ asymptotically because $\mathcal{D}_{o p t}^{d-1} \cong \frac{\pi^{2}}{4(d-1)^{2}} \cong \frac{\pi^{2}}{(2 d-1)^{2}}=\frac{\pi^{2}}{n^{2}}$.

In the case $n=2 d$, the initial state is expressed as $x_{\vec{a}_{d}^{\prime}}^{2 d} \stackrel{\text { def }}{=} \bigoplus_{k=0}^{d} a_{k} x_{E}^{2 k+1}$, where the vector $\vec{a}_{d}^{\prime}=\left(a_{k}\right)_{k=0}^{d}$ has no negative element. When we select a measurement similar to the one mentioned above, the estimation error is calculated to be $\frac{1}{2}\left(1-\sum_{k=0}^{d-1} a_{k} a_{k+1}\right)+\frac{1}{4} a_{0}$. Hence, the minimum error $\mathcal{D}_{\text {opt }}^{d}{ }^{\prime}$ satisfies $\mathcal{D}_{\text {opt }}^{d} \leq \mathcal{D}_{\text {opt }}^{d}{ }^{\prime} \leq \mathcal{D}_{\text {opt }}^{d-1}$. The same conclusion is obtained in the odd case.

Next, we investigate the reference space in the odd-dimensional case. When we use the above method, the dimension of the reference space $\mathcal{H}_{R, 2 d-1}$ is $2 d$. If the dimension of $\mathcal{H}_{2 d-1,2 k}$ is greater than that of $\mathcal{H}_{2 k}$, we can use the space $\mathcal{H}_{2 d-1,2 k}$ as the reference space. For $k=d$, the dimension of $\mathcal{H}_{2 d-1,2 k}$ is 1 , i.e., is smaller than that of $\mathcal{H}_{2 k}$. However, for $k<d$, the dimension of $\mathcal{H}_{2 d-1,2 k}$ is $\left(\begin{array}{c}2 d-1 \\ d-k\end{array}\right)-\left(\begin{array}{c}2 d-1 \\ d-k-1\end{array}\right)$, i.e., it is greater than that of $\mathcal{H}_{2 k}$. Hence, by replacing the reference space by the space $\mathcal{H}_{2 d-1,2 k}$, we can reduce the estimation error to $\mathcal{D}_{\text {opt }}^{d-2}$ without using the reference system. Since $\mathcal{D}_{\text {opt }}^{d-2} \cong \frac{\pi^{2}}{4(d-2)^{2}} \cong \frac{\pi^{2}}{(2 d-1)^{2}}=\frac{\pi^{2}}{n^{2}}$, 
an estimation error of $\frac{\pi^{2}}{n^{2}}$ can be achieved without the reference space. This discussion can also be applied to the case when $n$ is an even number.

\section{Technical details}

In the following, we investigate the derivations of equations (12) and (13). Since $\operatorname{Tr} V_{\hat{g}}^{j}$ and $d(I, \hat{g})$ depend only on the eigenvalues of $g e^{i \theta / 2}, e^{-i \theta / 2}$, the invariant distribution $\mu$ has the form $\mu(d \hat{g})=\frac{1}{\pi} \sin ^{2} \frac{\theta}{2} d \theta \sin \phi_{1} d \phi_{1} d \phi_{2}$ in the following parameterization:

$$
\hat{g}=\left(\begin{array}{cc}
\cos \phi_{1} & \sin \phi_{1} e^{i \phi_{2}} \\
-\sin \phi_{1} e^{-i \phi_{2}} & \cos \phi_{1}
\end{array}\right)^{\dagger}\left(\begin{array}{cc}
e^{i \theta / 2} & 0 \\
0 & e^{-i \theta / 2}
\end{array}\right)\left(\begin{array}{cc}
\cos \phi_{1} & \sin \phi_{1} e^{i \phi_{2}} \\
-\sin \phi_{1} e^{-i \phi_{2}} & \cos \phi_{1}
\end{array}\right)
$$

Hence, the relation

$$
\int_{\mathrm{SU}(2)} f(\theta) \mu(d \hat{g})=\int_{0}^{2 \pi} \frac{f(\theta)}{\pi} \sin ^{2} \frac{\theta}{2} d \theta
$$

holds. Because $d(I, \hat{g})=\sin ^{2} \frac{\theta}{2}$ and $\operatorname{Tr} V_{\hat{g}}^{j}=\sum_{l=1}^{j} e^{i\left(l-\frac{j+1}{2}\right) \theta}$ and by applying (15), we have

$$
\begin{aligned}
& \int_{\mathrm{SU}(2)} d(I, \hat{g})\left|\operatorname{Tr} V_{\hat{g}}^{j}\right|^{2} \mu(d \hat{g})=\int_{0}^{2 \pi} \frac{1}{\pi} \sin ^{4} \frac{\theta}{2}\left(\sum_{l=1}^{j} e^{i\left(l-\frac{j+1}{2}\right) \theta}\right) \overline{\left(\sum_{l=1}^{j} e^{i\left(l-\frac{j+1}{2}\right) \theta}\right)} d \theta \\
= & \int_{0}^{2 \pi} \frac{1}{4 \pi}\left(\frac{3}{2}-2 \cos \theta+\frac{1}{2} \cos 2 \theta\right)\left(j+2 \sum_{l=1}^{j}(j-l) \cos l \theta\right) d \theta .
\end{aligned}
$$

When $j \geq 2$, this integral is equal to

$$
\int_{0}^{2 \pi} \frac{1}{4 \pi}\left(\frac{3}{2}-2 \cos \theta+\frac{1}{2} \cos 2 \theta\right)(j+2(j-1) \cos \theta+2(j-2) \cos 2 \theta) d \theta
$$

which implies (12). When $j=1$, we have

$$
\int_{0}^{2 \pi} \frac{1}{4 \pi}\left(\frac{3}{2}-2 \cos \theta+\frac{1}{2} \cos 2 \theta\right) d \theta=\frac{1}{2}
$$

which implies (12). 
With regard to (13), we can calculate

$$
\begin{aligned}
& \int_{\mathrm{SU}(2)} d(I, \hat{g})\left(\operatorname{Tr} V_{\hat{g}}^{2 k}\right)\left(\operatorname{Tr} V_{\hat{g}}^{2 k^{\prime}}\right)^{\dagger} \mu(d \hat{g}) \\
= & \int_{0}^{2 \pi} \frac{1}{4 \pi}\left(\frac{3}{2}-2 \cos \theta+\frac{1}{2} \cos 2 \theta\right)\left(\sum_{l=1}^{2 k} e^{i\left(l-\frac{2 k+1}{2}\right) \theta}\right) \overline{\left(\sum_{l=1}^{2 k^{\prime}} e^{i\left(l-\frac{2 k^{\prime}+1}{2}\right) \theta}\right)} d \theta .
\end{aligned}
$$

In the above integral, the term $\left(\sum_{l=1}^{2 k} e^{i\left(l-\frac{2 k+1}{2}\right) \theta}\right) \overline{\left(\sum_{l=1}^{2 k^{\prime}} e^{i\left(l-\frac{2 k^{\prime}+1}{2}\right) \theta}\right)}$ can be expanded into many terms; however, all other terms except the constant term, $2 \cos \theta$, and $2 \cos 2 \theta$ vanish. That is, the coefficients of only the above three terms are important.

In the following, we calculate this integral in the three cases: (i) $k=k^{\prime}$, (ii) $\left|k-k^{\prime}\right|>1$, and (iii) $\left|k-k^{\prime}\right|=1$. Case (i) has already been calculated in (12). In case (ii), these three coefficients coincide. Thus, the integral is equal to 0 . Next, we proceed to the case (iii). When $k^{\prime}=k+1$, the term $\left(\sum_{l=1}^{2 k} e^{i\left(l-\frac{2 k+1}{2}\right) \theta}\right) \overline{\left(\sum_{l=1}^{2 k^{\prime}} e^{i\left(l-\frac{2 k^{\prime}+1}{2}\right) \theta}\right)}$ can be expanded to $k+2 k \cos \theta+2(k-$ $1) \cos 2 \theta+\cdots$. Thus, the integral is equal to $-\frac{1}{4}$. Similarly, we can show that the integral is equal to $-\frac{1}{4}$ when $k^{\prime}=k-1$. Therefore, we obtain (13).

\section{Concluding remark}

In this paper, we derived a remarkable relation between the estimation of the $\mathrm{SU}(2)$ action and phase estimation. By using this relation, we showed that the optimal estimation error is less than $\frac{\pi^{2}}{n^{2}}$. The essence of this relation lies in the relation between the two similar relations (7) and (13). Indeed, since $\sin ^{2} \theta=1-\left|\frac{e^{i \theta / 2}+e^{-i \theta / 2}}{2}\right|^{2}$, the cost function of phase estimation is equal to $1-\left|\chi_{2}(\theta-\hat{\theta})\right|^{2}$, where $\chi_{2}(\theta)$ is the half of the character of the two-dimensional representation $e^{i \theta} \mapsto\left(\begin{array}{cc}e^{i \theta / 2} & 0 \\ 0 & e^{-i \theta / 2}\end{array}\right)$. Hence, the essence of $(7)$ is the equation

$$
\int_{0}^{2 \pi}\left(1-\left|\chi_{2}(\theta)\right|^{2}\right) \chi^{k}(\theta) \overline{\chi^{l}(\theta)} d \theta=\frac{1}{2} \delta_{k, l}-\frac{1}{4} \delta_{k, l-1}-\frac{1}{4} \delta_{k-1, l}
$$

where $\chi^{l}(\theta)$ is the character of the one-dimensional representation of $e^{i \theta} \mapsto e^{i l \theta}$.

On the other hand, when we let $\chi^{l}(g)$ assume the character of the $l$-dimensional irreducible representation of $\mathrm{SU}(2)$ over dimension $l$, the essence of (13) is

$$
\int_{\mathrm{SU}(2)}\left(1-\left|\chi^{2}(g)\right|^{2}\right) \chi^{2 k}(g) \overline{\chi^{2 l}(g)} \mu(d g)=\frac{1}{2} \delta_{k, l}-\frac{1}{4} \delta_{k, l-1}-\frac{1}{4} \delta_{k-1, l} .
$$


Therefore, the main reason for the relation between the two estimation problems is the character formulas (17) and (16). In other words, the integrals with regard to characters are the essence of phase estimation as well as that of the estimation of $\mathrm{SU}(2)$ action. Physically, the square-error estimators in both scenarios can be realized by the effective use of interference between different irreducible representations.

In this paper, when we consider the irreducible space $\mathcal{H}_{n, k}$ of the action of the permutation group as the reference space of the irreducible space $\mathcal{H}_{k}$ of $\mathrm{SU}(2)$ action, we proved the existence of a square-error estimator in the estimation of the $\mathrm{SU}(2)$ action without use of the real reference system. Since the irreducible space $\mathcal{H}_{n, k}$ of the action of the permutation group has a greater dimension than the corresponding irreducible space $\mathcal{H}_{k}$ of the $\mathrm{SU}(2)$ action, except for the exceptional cases, the correspondence between the irreducible space $\mathcal{H}_{n, k}$ and the reference space of the corresponding irreducible space $\mathcal{H}_{k}$ is not unique. Hence, it is desirable to obtain a more physically realizable correspondence[13]. This is an interesting future study.

Finally, we should remark on the relation between our group covariance approach and the Cramér-Rao approach. In the latter approach, we focus on the Cramér-Rao-type bound, i.e., the minimum weighted sum of mean square errors under locally unbiased conditions. As discussed in Fujiwara and Imai[7], we often seek an input state that minimizes this bound. Even though this bound decrease to zero with an order of $\frac{1}{n^{2}}$, we cannot conclude that there exists a sequence of estimators whose weighted sum of minimum mean square errors decreases to zero with an order of $\frac{1}{n^{2}}$. In the state estimation, there exists a sequence of estimators whose weighted sum of minimum mean square errors reduces to zero at rate $\frac{C}{n}$, where $C$ is the Cramér-Rao-type bound. This is because an adaptive estimator attaining bound $C$ can be selected as follows $[8,9]$. In the state estimation, we can select a set of neighborhoods in which the minimum weighted sum of mean square errors can be approximated to the Cramér-Rao type bounds within bounded differences. Hence, if we choose the first estimator whose estimate belongs to the above neighborhood with an exponentially small error probability, the adaptive estimator satisfies the required condition. However, the situation of the estimation of the $\mathrm{SU}(2)$ action differs from that of the estimation of state. In the estimation of the $\mathrm{SU}(2)$ action, such neighborhoods depend on the number $n$ because the state family depends on the input state and the required neighborhoods depend on the state family. In other words, there is a possibility that the radius of the neighborhoods reduces to zero in proportion with the number $n$ of actions. Hence, we cannot directly obtain the estimation error based on the optimal Cramér-Rao-type bound.

On completion of this research, the author found that the same results were obtained by two other groups[10,11,12]. However, this approach is different 
from those employed by them because it is based on the notable relation between the $\mathrm{SU}(2)$ estimation and the phase estimation. Further, Chiribella

et al. [16] proved the optimality of the error $\frac{1}{2}\left(1-\sum_{k=1}^{d-1}\left|x_{k}\right|\left|x_{k+1}\right|\right)$ while this is not proved in this paper. They also elucidated out that character integrals and interference of irreducible representations are very useful for finding the optimal estimation of an unknown group transformation not only for $U(1)$ and $\mathrm{SU}(2)$ but also for arbitrary groups.

Further, on completion of this paper, the author also found Acin et al.'s paper [15]. They almost mentioned that the optimal initial state has the form $x_{\vec{x}_{d}}^{2 d-1}$. However, they did not derive the equation corresponding to (14). In addition, Rudolph and Grover [14] discussed a similar problem from a computational viewpoint.

\section{Acknowledgments}

The author wishes to thank Professor Keiji Matsumoto, Dr. Yoshiyuki Tsuda, and Mr. Aram W. Harrow for their useful discussions. The author is also grateful to Professor Hiroshi Imai and all the members of ERATO Quantum Computation and Information Project for their kind support. The author would also like to thank Dr. Terry Rudolph for kind comments for concluding remark. The author is indebted to the reviewer for some useful comments.

\section{References}

[1] V. Bužek, R. Derka, and S. Massar, "Optimal Quantum Clocks," Phys. Rev. Lett. 82, 2207, 1999; quant-ph/9808042.

[2] A. Fujiwara, "Estimation of SU(2) operation and dense coding: An information geometric approach," Phys. Rev. A 65, 012316 (2002).

[3] M. A. Ballester, "Estimation of unitary quantum operations," Phys. Rev. A, 69, 022303 (2004); quant-ph/0305104.

[4] C. W. Helstrom, Found. of Phys., 4, 453 (1974).

[5] A.S. Holevo, "Covariant measurements and uncertainty relations," Rep. Math. Phys., 16, 385-400, (1979).

[6] A. S. Holevo, Probabilistic and Statistical Aspects of Quantum Theory, (NorthHolland, Amsterdam, 1982). Originally published in Russian (1980).

[7] A. Fujiwara and H. Imai, "Quantum parameter estimation of a generalized Pauli channel," J. Phys. A: Math. and Gen., 36, 8093-8103, (2003). 
[8] R. Gill and S. Massar, "State estimation for large ensembles," Phys. Rev. A, 61, 042312 (2000).

[9] M. Hayashi and K. Matsumoto, "Statistical model with measurement degree of freedom and quantum physics," Chapter 13: Measurement of Qubits, Collected Papers in Asymptotic Theory of Quantum Statistical Inference: Selected papers. Ed: Masahito Hayashi (World Scientific Press, 2005). Originally published in Japanese as Surikaiseki Kenkyuusho Kokyuroku, 1055, 96-110 (1998).

[10] E. Bagan, M. Baig, and R. Muñoz-Tapia, "Entanglement-assisted alignment of reference frames using a dense covariant coding," Phys. Rev. A, 69, 050303(R) (2004); quant-ph/0303019.

[11] E. Bagan, M. Baig, and R. Muñoz-Tapia, "Quantum reverse-engineering and reference-frame alignment without nonlocal correlations," Phys. Rev. A, 70, 030301(R) (2004); quant-ph/0405082.

[12] G. Chiribella, G. M. D'Ariano, P. Perinotti, and M. F. Sacchi, "Efficient use of quantum resources for the transmission of a reference frame," Phys. Rev. Lett., 93, 180503 (2004); quant-ph/0405095

[13] T. Rudolph, private communication (2004).

$[14]=\mathrm{T}$. Rudolph, and L. Grover, "Quantum Communication Complexity of Establishing a Shared Reference Frame," Phys. Rev. Lett., 91, 217905 (2003); quant-ph/0306017

[15] A. Acín, E. Jané, and G. Vidal, "Optimal estimation of quantum dynamics," Phys. Rev. A, 64, 050302 (2001); quant-ph/0012015

[16] G. Chiribella, G. M. D'Ariano, and M. F. Sacchi, "Optimal estimation of group transformations using entanglement," Phys. Rev. A, 72, 042338 (2005). 